\title{
Polymer translocation through a nanopore assisted by an environment of active rods
}

\author{
Hamidreza Khalilian, ${ }^{1, *}$ Jalal Sarabadani $\odot,{ }^{1, \dagger}$ and Tapio Ala-Nissila $\odot^{2,3}$ \\ ${ }^{1}$ School of Nano Science, Institute for Research in Fundamental Sciences (IPM), 19395-5531 Tehran, Tehran, Iran \\ ${ }^{2}$ Department of Applied Physics and QTF Center of Excellence, Aalto University, P.O. Box 11000, 00076 Aalto, Espoo, Finland \\ ${ }^{3}$ Interdisciplinary Centre for Mathematical Modelling and Department of Mathematical Sciences, \\ Loughborough University, Loughborough, Leicestershire LE11 3TU, United Kingdom
}

(Received 7 November 2020; accepted 7 January 2021; published 26 January 2021)

\begin{abstract}
We use a combination of computer simulations and isoflux tension propagation (IFTP) theory to investigate the translocation dynamics of a flexible linear polymer through a nanopore into an environment composed of repulsive active rods in two dimensions. We demonstrate that the rod activity induces a crowding effect on the polymer, leading to a time-dependent effective net force that facilitates translocation into the active environment. Incorporating this force into the IFTP theory for pore-driven translocation allows us to characterize translocation dynamics in detail and derive a scaling form for the average translocation time as $\tilde{\tau} \sim N_{01}^{1+v} \tilde{L}_{\mathrm{r}}^{v} / \tilde{F}_{\mathrm{SP}}$, where $N_{01}$, $\tilde{L}_{\mathrm{r}}$, and $\tilde{F}_{\mathrm{SP}}$ are the initial contour length of the $c i s$-side subchain, rod length, and self-propelling force acting on the rods, respectively, and $v$ is the equilibrium Flory scaling exponent.
\end{abstract}

DOI: 10.1103/PhysRevResearch.3.013080

\section{INTRODUCTION}

Translocation dynamics of biopolymers through nanopores has been one of the most active research areas in soft matter during the past few decades (see, e.g., Refs. [1-4] and references therein). The most relevant examples include DNA and mRNA translocation through nuclear pores, protein transportation across a membrane, and DNA injection by a virus. There are many applications from DNA sequencing to gene therapy and controlled drug delivery [5], and forced translocation was originally suggested as an inexpensive and fast method for DNA sequencing. Motivated by these applications, many experimental as well as theoretical works [6-41] have been performed since the seminal works by Bezrukov et al. [42] and by Kasianowicz et al. [43]. To date, most studies have focused on the dynamics of polymer translocation facilitated by external driving in the pore or by pulling the polymer from the head bead by optical tweezers, both of which are experimentally feasible [20-30].

In biological systems, however, polymer translocation processes often occur in crowded environments [5]. Such environments may be composed of diffusive and randomly distributed spherical static obstacles [44-47] or chaperones that assist transport across membranes [48-50]. Crowded environments consisting of active particles (APs) have introduced a new out-of-equilibrium-dynamics field of research with rich physics [51,52]. Examples include synthetic motile objects from molecular scale to microns [53,54], micro-

\footnotetext{
*khalilian@ipm.ir

$\dagger$ jalal@ipm.ir

Published by the American Physical Society under the terms of the Creative Commons Attribution 4.0 International license. Further distribution of this work must maintain attribution to the author(s) and the published article's title, journal citation, and DOI.
}

scopic living organisms [55,56], and artificial swimmers from nanometer to millimeter scales [57-69]. The presence of APs has a strong influence on polymer chains in equilibrium and can lead to significant conformational and dynamical changes (collapse or swelling) depending on the system parameters [70-76].

An interesting open question pertains to the influence of APs on polymer translocation dynamics. Pore-driven polymer translocation in the presence of spherical APs has been considered using computer simulation methods [77] and it was found that with high activity, there is a crowding effect in two dimensions (2D) that leads to a speed-up of translocation. An interesting and unanswered question remains concerning the effect of the APs on the dynamics of unforced or nondriven translocation. In many cases active objects such as bacteria are not spherical, but assume rodlike shapes. The combination of anisotropy in the shape of the APs and the presence of a self-propelling (SP) force that makes the objects active leads to interesting collective dynamics. In the case of rodlike APs, there is orientational alignment of the active rods (ARs) along the walls in a two-dimensional confining channel [78] and also in their collective motion [79]. The presence of a translocating polymer in such an environment thus warrants closer examination.

To this end, we perform here extensive computer simulations of unforced polymer translocation dynamics in the presence of ARs on the trans side of the pore. We demonstrate that when the chain is initially placed in such a way that a part of the chain is in the trans compartment, the presence of ARs induces a net effective force from the cis to the trans side that overcomes entropic forces and facilitates translocation. The presence of a time-dependent driving force allows us to use the isoflux tension propagation (IFTP) theory, which is benchmarked against the simulation data. As our main theoretical result, we find that the mean translocation time $\tilde{\tau}$ scales with the initial contour length of the cis-side subchain $N_{01}$, rod length $\tilde{L}_{\mathrm{r}}$, and SP force $\tilde{F}_{\mathrm{SP}}$ as $\tilde{\tau} \sim N_{01}^{1+v} \tilde{L}_{\mathrm{r}}^{v} / \tilde{F}_{\mathrm{SP}}$, where $v$ is the 

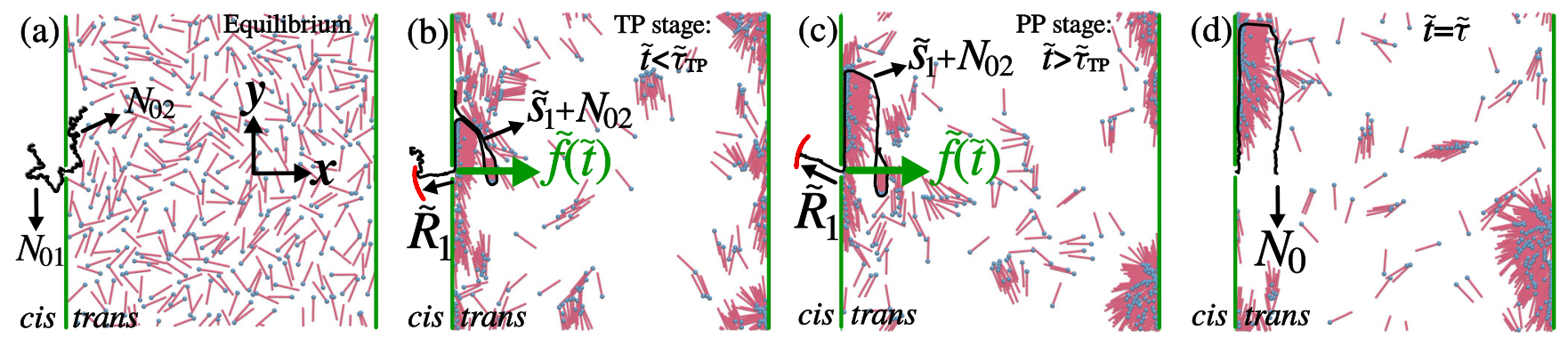

FIG. 1. (a) Configuration of the system after equilibration with passive rods with the chain fixed inside the pore. The compartment dimensions are $L_{x}=2 L_{y}=400$ (equally partitioned by a membrane in the $y$ direction), $L_{\mathrm{r}}=16$ (16 beads), and the number of rods $N_{\mathrm{r}}=320$, which gives a number density of 0.128 . Translocation is initiated from this state at $\tilde{t}=0$ by making the rods active with $F_{\mathrm{SP}}=32$ and releasing the chain. Here $N_{01}$ and $N_{02}$ are the initial contour lengths of the cis-and trans-side subchains, respectively. (b) Snapshot of the system during the tension propagation (TP) stage, i.e., $\tilde{t}<\tilde{\tau}_{\mathrm{TP}}$. The location of the tension front is denoted by $\tilde{R}_{1}$, and $\tilde{s}_{1}+N_{02}$ is the translocation coordinate (the number of monomers on the trans side). The SP force $F_{\mathrm{SP}}$ acts on the blue head monomer of each rod and is directed parallel to its axis from tail to head. The effective force imposed by the rods on the chain $\tilde{f}(\tilde{t})$ originates from the interaction between the active rods and the trans-side subchain. (c) Same as in (b) but for the postpropagation stage, i.e., $\tilde{t}>\tilde{\tau}_{\mathrm{TP}}$, where the tension has already reached the $c i s$-side subchain end. (d) Final configuration of the system at the end of the translocation process.

equilibrium Flory exponent. This scaling form for the translocation time is valid in the limit of long chains $\left(N_{01} \gg 1\right)$ and its dependence on $\tilde{L}_{\mathrm{r}}$ and $\tilde{F}_{\mathrm{SP}}$ comes from the effective net force acting on the monomer(s) at the pore induced by the APs with the trans-side subchain. This leads to scaling of $\tilde{\tau}$ with $N_{01}$ identical to that of a pore-driven chain without APs.

The structure of the paper is as follows. In Sec. II we present details of the Langevin dynamics (LD) computer simulations for a coarse-grained model of active rods and a translocating polymer chain. The IFTP theory is introduced in Sec. III. Section IV is devoted to presentation and discussion of our main results. A summary and conclusions are in Sec. V.

\section{LANGEVIN DYNAMICS SIMULATIONS}

Our system comprises a flexible, self-avoiding polymer chain of length $N_{0}$ modeled by the bead-spring model [80] with beads having a pairwise shifted repulsive Lennard-Jones (SRLJ) interaction $U_{\mathrm{LJ}}(r)=4 \varepsilon\left[\left(\frac{\sigma}{r}\right)^{12}-\left(\frac{\sigma}{r}\right)^{6}\right]+\varepsilon$ if $r \leqslant 2^{1 / 6}$ and zero otherwise. Here $\sigma$ is the LJ radius, $\varepsilon$ is the potential well depth, and $r$ is the distance between two monomers. In addition, the consecutive monomers are connected by the finitely extensible nonlinear elastic interaction $U_{\mathrm{FENE}}(r)=$ $-\frac{1}{2} k R_{0}^{2} \ln \left[1-r^{2} / R_{0}^{2}\right]$, where $k$ and $R_{0}$ are the string constant and the maximum allowed distance between the consecutive monomers, respectively. The polymer is put into a container of size $L_{x}=2 L_{y}=400$ in units of $\sigma$ and there is a membrane in the middle (see Fig. 1) with a nanopore of radius $1.5 \sigma$ allowing only one bead in the pore at a time. The container has walls in the $x$ direction and periodic boundary conditions in the $y$ direction, and its walls and the membrane interact with the chain with the same SRLJ potential. In the trans side there are $N_{\mathrm{r}}$ rigid rods comprising SRLJ beads of radius $\sigma$ whose length is $L_{\mathrm{r}} \sigma$. To model self-propulsion, a SP force with magnitude of $F_{\mathrm{SP}}$ is added to the head bead of each rod along its main axis and from its tail to its head.

For the simulations we employ LD, where for the position of the $i$ th monomer of the polymer $M \ddot{\vec{r}}_{i}=-\eta \dot{\vec{r}}_{i}-\vec{\nabla} U_{\mathrm{m} i}+$ $\vec{\xi}_{i}(t)$. Here $\eta$ is the friction coefficient, $U_{\mathrm{m} i}$ is the sum of all interactions, and $\vec{\xi}_{i}$ is white noise with $\left\langle\vec{\xi}_{i}(t)\right\rangle=0$ and $\left\langle\vec{\xi}_{i}(t) \vec{\xi}_{j}\left(t^{\prime}\right)\right\rangle=2 \eta k_{\mathrm{B}} T \delta_{i j} \delta\left(t-t^{\prime}\right)$, where $k_{\mathrm{B}}$ is the Boltzmann constant, $T$ is the temperature, and $\delta_{i j}$ and $\delta\left(t-t^{\prime}\right)$ are the Kronecker and Dirac delta functions, respectively. For the $i$ th bead of each rod we add the SP force as $M \ddot{\vec{r}}_{i}=-\eta \dot{\vec{r}}_{i}+$ $F_{\mathrm{SP}} \delta_{i h} \hat{\mathbf{e}}-\vec{\nabla} U_{\mathrm{r} i}+\vec{\xi}_{i}(t)$, where $h$ picks the head bead, $\hat{\mathbf{e}}$ is the unit vector parallel to the vector connecting the tail to the head, and $U_{\mathrm{r} i}$ is the sum of all interactions on the $i$ th bead. We use $M, \sigma$, and $\varepsilon$ as the units for mass, length, and energy, respectively, where $M=1$ is the mass of each monomer in the polymer and the rods and $\varepsilon=1$. The temperature is kept at $k_{\mathrm{B}} T=1.2$, the solvent friction coefficient is $\eta=0.7$, and $\tau_{0}=\sqrt{M \sigma^{2} / \varepsilon}$ is the simulation time unit. In our simulations, the integration time step is $d t=0.001 \tau_{0}$. Finally, the spring constant is set to $k=30$ and $R_{0}=1.5$. The simulations were performed using the LAMMPS [81] package.

Before the translocation process, the polymer is fixed in the pore such that there are $N_{01}$ and $N_{02}$ beads in the $c i s$ and trans compartments, respectively, where $N_{0}=1+N_{01}+N_{02}$. The polymer-rod system is equilibrated for $t_{\mathrm{eq}}=5 \times 10^{4} \tau_{0}$ without the SP force, and at the beginning of translocation the polymer is released and $F_{\mathrm{SP}}$ is turned on for all rods simultaneously. The rod lengths used in the LD simulations are $L_{\mathrm{r}}=8$, 12,16 , and 20 , which correspond to the number of rods in the trans domain $N_{\mathrm{r}}=640,426,320$, and 256, respectively. For the SP force we have $F_{\mathrm{SP}}=16,24$, and 32, the initial cis-side contour lengths are $N_{01}=50,100$, and 150 , and finally the initial trans-side contour length is $N_{02}=100$. For all sets of parameters the value of the rods' volume fraction is fixed at about 0.1 in 2D. The density of the ARs here are chosen low enough such that the equilibrated system has a uniformly random orientational distribution. For the particular system with $N_{01}=N_{02}=100, \tilde{L}_{\mathrm{r}}=16$, and $\tilde{F}_{\mathrm{SP}}=32$ the results are averaged over 3000 uncorrelated trajectories, while for the other cases we use at least 1000 trajectories.

We use dimensionless quantities throughout and a tilde denotes units within the IFTP theory. They are defined as $\tilde{Q} \equiv$ $Q / Q_{u}$, where the denominator denotes the units of length, time, velocity, monomer flux, friction, and force as $s_{u} \equiv \sigma$, $t_{u} \equiv \eta \sigma^{2} /\left(k_{\mathrm{B}} T\right), v_{u} \equiv \sigma / t_{u}=k_{\mathrm{B}} T /(\eta \sigma), \phi_{u} \equiv k_{\mathrm{B}} T /\left(\eta \sigma^{2}\right)$, $\Gamma_{u} \equiv \eta$, and $f_{u} \equiv k_{\mathrm{B}} T / \sigma$, respectively. 

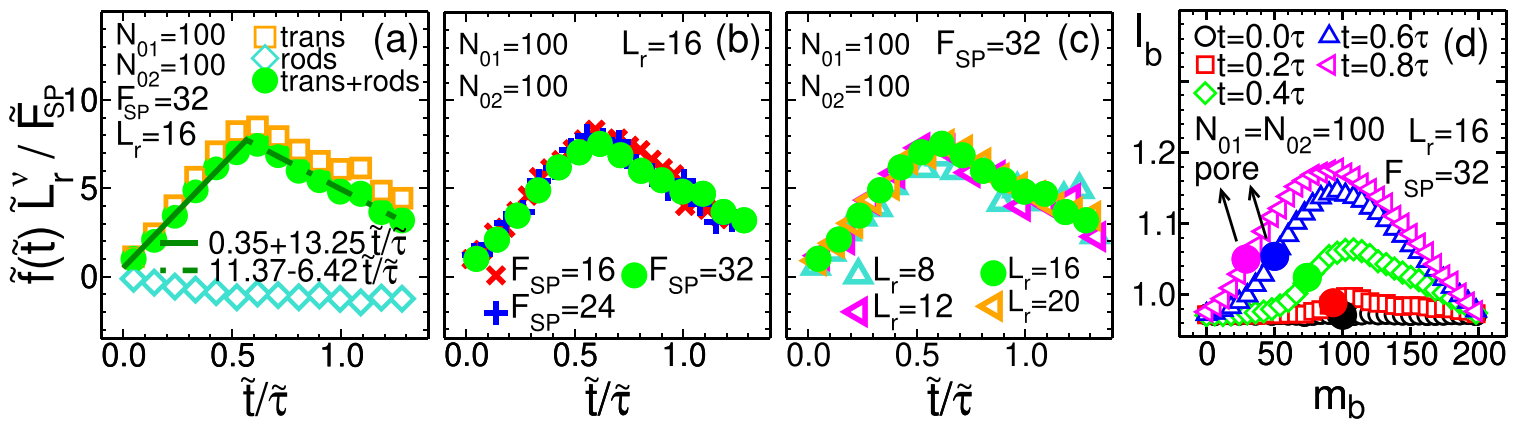

FIG. 2. (a) Plot of $\tilde{f}(\tilde{t}) \tilde{L}_{\mathrm{r}}^{v} / \tilde{F}_{\mathrm{SP}}$ as a function of the normalized time $\tilde{t} / \tilde{\tau}$, where $\tilde{f}(\tilde{t})$ is the effective force experienced by the monomer inside the pore in the $x$ direction from the LD simulations, $v$ is the Flory exponent, and $\tilde{\tau}$ is the translocation time (as obtained from LD) for $L_{\mathrm{r}}=16, N_{\mathrm{r}}=320$, and $F_{\mathrm{SP}}=32$. Open orange squares and open turquoise diamonds are data for the contributions of the tension due to the trans-side subchain and the interactions of ARs with the monomer inside the pore, respectively. The effective force which is the sum of the tension due to the trans-side subchain and the AR interactions with the monomer inside the pore is given by green circles. The solid and the dashed green lines are the fitting curves for the effective force in the TP and PP stages, respectively. Here $N_{01}=100$ and $N_{02}=100$ are the initial contour lengths of the cis- and trans-side subchains, respectively. (b) Same as in (a) but for fixed $L_{\mathrm{r}}=16$ and for different values of $F_{\mathrm{SP}}=16,24$, and 32. (c) Same as in (b) but for fixed $F_{\mathrm{SP}}=32$ and different values of $L_{\mathrm{r}}=8,12,16$, and 20 corresponding to the number of rods $N_{\mathrm{r}}=640,426,320$, and 256, respectively. (d) Bond length $l_{\mathrm{b}}$ as a function of the bond index $m_{\mathrm{b}}$ with the same system parameters as in (a) for different time instants during the translocation process $t / \tau=0-0.8$. The bond indices $m_{\mathrm{b}}=1$ and 200 identify the tail and head bonds in the cis and trans sides, respectively.

Figure 1(a) presents a typical snapshot of the system after equilibration at $\tilde{t}=0$. We find that the activity of the rods induces an effective force $\tilde{f}(\tilde{t})$ on the monomer in the pore, directed from cis to trans such that it facilitates translocation as depicted in Fig. 1(b). This causes tension propagation along the backbone of the cis-side subchain. The distance between the tension front on the cis side from the pore is denoted by $\tilde{R}_{1}$. This stage is called the tension propagation (TP) stage $(\tilde{t}<$ $\left.\tilde{t}_{\mathrm{TP}}\right)$. The number of the monomers on the trans side $\tilde{s}_{1}+N_{02}$ defines a translocation coordinate and is equal to $N_{02}$ at time zero. Eventually the tension reaches the end of the cis-side subchain and the postpropagation (PP) stage starts $\left(\tilde{t}>\tilde{t}_{\mathrm{TP}}\right)$, which is presented in Fig. 1(c). Finally, Fig. 1(d) shows the last snapshot of the system at the end of the translocation process which defines the translocation time $\tilde{\tau}$.

\section{ISOFLUX TENSION PROPAGATION THEORY}

Over the past few years a consistent and quantitatively accurate theory of driven polymer translocation has been developed based on isoflux tension propagation. Due to the effective force $\tilde{f}(\tilde{t})$ induced by the SP forces of the rods, we can generalize the IFTP theory to the present case too. To obtain the time evolution of the translocation coordinate $\tilde{s}_{1}$, which is the number of monomers that have passed from the cis to the trans side, the isoflux approximation for the monomer flux $\tilde{\phi}_{1}(\tilde{t})=d \tilde{s}_{1} / d \tilde{t}$ means that it is constant in space but evolves in time within the mobile subchain on the cis side. The tension force on the backbone of the chain at distance $\tilde{x}$ from the pore on the cis side $\tilde{\mathcal{F}}(\tilde{x}, \tilde{t})$ is obtained by integration of the local tension force balance relation $d \tilde{\mathcal{F}}\left(\tilde{x}^{\prime}, \tilde{t}\right)=-\tilde{\phi}_{1}(\tilde{t}) d x^{\prime}$ from the pore entrance at $\tilde{x}^{\prime}=0$ to the distance $\tilde{x}$ as $\tilde{\mathcal{F}}(\tilde{x}, \tilde{t})=\tilde{\mathcal{F}}_{0}-$ $\tilde{x} \tilde{\phi}_{1}(\tilde{t})$ [here the tension force at the entrance of the pore on the cis side is $\left.\tilde{\mathcal{F}}_{0}=\tilde{f}(\tilde{t})-\tilde{\eta}_{\mathrm{p}} \tilde{\phi}_{1}(\tilde{t})\right]$. Then, as the tension force vanishes at $\tilde{R}_{1}$, i.e., $\tilde{\mathcal{F}}\left(\tilde{R}_{1}, \tilde{t}\right)=0$, one can write $\tilde{\mathcal{F}}_{0}=\tilde{R}_{1} \tilde{\phi}_{1}(\tilde{t})$. Finally, using the above definitions of the monomer flux and $\tilde{\mathcal{F}}_{0}$, the equation of motion for the translocation coordinates is cast in the form $[26,27]$

$$
\tilde{\Gamma}_{1}(\tilde{t}) \frac{d \tilde{s}_{1}}{d \tilde{t}}=\tilde{f}(\tilde{t}),
$$

where $\tilde{\Gamma}_{1}(\tilde{t})=\tilde{R}_{1}(\tilde{t})+\tilde{\eta}_{\mathrm{p}}$, with $\tilde{\eta}_{\mathrm{p}}$ and $\tilde{R}_{1}(\tilde{t})$ the pore friction and the tension front distance from the nanopore, respectively.

In Fig. 2(a) we plot $\tilde{f}(\tilde{t}) \tilde{L}_{\mathrm{r}}^{v} / \tilde{F}_{\mathrm{SP}}$, where $\tilde{f}(\tilde{t})$ is the effective force (closed green circles) (is the sum of the tension due to the trans-side subchain and the AR interactions with the monomer inside the pore) in the horizontal direction from cis to trans as a function of the normalized time $\tilde{t} / \tilde{\tau}$ for $N_{01}=N_{02}=100, F_{\mathrm{SP}}=32$, and $L_{\mathrm{r}}=16$. It is the sum of the tension force due to the trans-side subchain (open orange squares) and the force due to the interactions of the ARs with the monomer inside the pore (open turquoise diamonds). The effective force first grows almost linearly and then decreases. Its largest magnitude in $\mathrm{LJ}$ units is of the same order as that of $F_{\mathrm{SP}}$. In Figs. 2(b) and 2(c) we show the corresponding data for varying either $F_{\mathrm{SP}}$ or $L_{\mathrm{r}}$. The remarkable finding here is that all the data collapse onto two master curves, namely, $\tilde{f}_{\mathrm{TP}}(\tilde{t}) \tilde{L}_{\mathfrak{x}}^{v} / \tilde{F}_{\mathrm{SP}}=0.35+13.25 \tilde{t} / \tilde{\tau}$ [solid green line in Fig. 2(a)] and $\tilde{f}_{\mathrm{PP}}(\tilde{t}) \tilde{L}_{\mathrm{r}}^{v} / \tilde{F}_{\mathrm{SP}}=11.37-6.42 \tilde{t} / \tilde{\tau}$ [dash-dotted green line in Fig. 2(a)] in the TP and PP stages, respectively, and they intersect at $\tilde{t} / \tilde{\tau}=0.56$. We have independently verified from the bond lengths that the maximum of the effective force curve exactly corresponds to the TP time where the tension front reaches the end of the cis-side subchain. To this end, in Fig. 2(d) we plot the bond length $\mathrm{t}_{\mathrm{b}}$ as a function of the bond index $m_{\mathrm{b}}$. The bond indices $m_{\mathrm{b}}=1$ and 200 identify the tail and head bonds on the cis and trans sides, respectively. It can be seen from the figure that the tension front reaches the tail bond $\left(m_{\mathrm{b}}=1\right)$ at about $\tilde{t} / \tilde{\tau}=0.56 \approx 0.6$ (open blue triangles), which defines the TP time. We have checked this for all the sets of parameters here.

Solving Eq. (1) gives the translocation coordinate $\tilde{s}_{1}$ provided that the time evolution of the location of the tension front $\tilde{R}_{1}$ is known. Using the end-to-end distance $\tilde{R}_{\text {le }}$ 


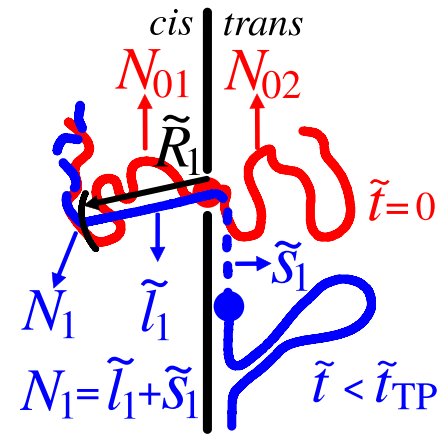

FIG. 3. Schematic of polymer configurations at time zero (in red) and at time $\tilde{t}<\tilde{t}_{\mathrm{TP}}$ (in blue), where $\tilde{t}_{\mathrm{TP}}$ denotes the tension propagation time. For the sake of clarity, the active rods in the trans side have not been depicted. The initial contour lengths of the cisand trans-side subchains at time zero are $N_{01}$ and $N_{02}$, respectively, and the polymer is in its equilibrium state while the monomer (closed red circle) that identifies the border between the initial cis- and trans-side subchains is fixed at the pore. At time $\tilde{t}, \tilde{s}_{1}$ is the number of monomers that was initially in the cis side and has traversed the pore (dotted blue line), $\tilde{l}_{1}$ is the number of mobile monomers in the cis side (solid blue line in the cis side), and the equilibrium part of the subchain in the cis side is the dashed blue line. Here $N_{1}$ is the number of all monomers that have experienced the tension force by time $\tilde{t}$ and thus $N_{1}=\tilde{l}_{1}+\tilde{s}_{1}$. The closed blue circle separates the initial cis-side subchain from the trans-side side subchain. The location of the tension front is shown by $\tilde{R}_{1}$.

corresponding to the extent of the mobile part of the cis-side subchain at time zero just before the translocation process, wherein the chain is in its equilibrium state, the location of the tension front $\tilde{R}_{1}$ and its equation of motion can be obtained. To understand why $\tilde{R}_{1}$ is equal to $\tilde{R}_{1 \mathrm{e}}$ we just need to compare the $c i s$-side subchain configurations at time zero with the ones at time $\tilde{t}$ as depicted in Fig. 3. For visualization purposes, in Fig. 3 the polymer configurations at time zero (in red) and at time $\tilde{t}<\tilde{t}_{\mathrm{TP}}$ (in blue) are depicted, where $\tilde{t}_{\mathrm{TP}}$ denotes the tension propagation time. For the sake of clarity, the active rods on the trans side are not depicted. The initial contour lengths of the cis- and trans-side subchains at time zero are $N_{01}$ and $N_{02}$, respectively, and the polymer is in its equilibrium state while the monomer (closed red circle) that identifies the border between the initial cis- and trans-side subchains is fixed at the pore. As time passes, at time $\tilde{t}$ a part of the subchain that was initially in the cis side has traversed the pore (dotted blue line) and $\tilde{s}_{1}$ monomers have gone through. At time $\tilde{t}, \tilde{l}_{1}$ is the number of mobile monomers on the cis side (solid blue line in the cis side) and the equilibrium part of the subchain in the cis side is the dashed blue line. In addition, $N_{1}$ is the number of all monomers that have experienced the tension force by time $\tilde{t}$ and thus $N_{1}=\tilde{l}_{1}+\tilde{s}_{1}$. The closed blue circle separates the initial cis-side subchain from the trans-side subchain. The location of the tension front is shown by $\tilde{R}_{1}$. As mentioned above, the number of monomers affected by the tension front on the cis side at time $\tilde{t}$ is $N_{1}$. On the other hand, at time zero these $N_{1}$ monomers are accommodated in the space between the nanopore and the current location of the tension front at time $\tilde{t}$. The linear size of this accommodating space, on the one hand, at time zero is given by the end-to-end distance of the $N_{1}$ monomers as $\tilde{R}_{1 \mathrm{e}}=A_{v} N_{1}^{v}$ and, on the other hand, at time $\tilde{t}$ it is identified as the distance between the tension front and the nanopore $\tilde{R}_{1}$. Therefore, $\tilde{R}_{1}=\tilde{R}_{1 \mathrm{e}}=A_{v} N_{1}^{v}$. Moreover, as $N_{1}=\tilde{l}_{1}+\tilde{s}_{1}$, where $\tilde{l}_{1}$ is the number of monomers in the mobile domain (affected by the tension front) on the cis side and $\tilde{s}_{1}$ is the translocation coordinate as defined above, we can simply write $\tilde{R}_{1}=A_{v}\left(\tilde{l}_{1}+\tilde{s}_{1}\right)^{v}$. Here $A_{v}=1.1$ for the present LD model and $v=3 / 4$ is the equilibrium Flory scaling exponent in 2D. Assuming that the mobile part of the cis-side subchain is fully straightened corresponding to the strong stretching (SS) regime of polymer translocation dynamics, we can write $\tilde{l}_{1}=\tilde{R}_{1}[19,24]$. Together with the definition of the monomer flux $\tilde{\phi}_{1}=d \tilde{s}_{1} / d \tilde{t}$ and differentiating both sides of $\tilde{R}_{1}=A_{\nu}\left(\tilde{R}_{1}+\tilde{s}_{1}\right)^{v}$ in time, the equation of motion for $\tilde{R}_{1}$ in the TP stage is

$$
\dot{\tilde{R}}_{1}(\tilde{t})=\frac{\nu A_{v}^{1 / \nu} \tilde{R}_{1}(\tilde{t})^{(\nu-1) / v} \tilde{\phi}_{1}(\tilde{t})}{1-v A_{v}^{1 / v} \tilde{R}_{1}(\tilde{t})^{(\nu-1) / v}} .
$$

In the PP stage the tension has already reached the cisside subchain. Differentiating the closure $N_{1}=\tilde{l}_{1}+\tilde{s}_{1}=N_{01}$ gives the time evolution of $\tilde{R}_{1}$ as

$$
\dot{\tilde{R}}_{1}(\tilde{t})=-\tilde{\phi}_{1} .
$$

To have the full solution of the IFTP theory in the TP stage both Eqs. (1) and (2) must be self-consistently solved, while in the PP stage one has to solve Eqs. (1) and (3).

\section{RESULTS}

\section{A. Waiting time distribution}

To validate the IFTP theory it is useful to investigate the waiting time (WT) distribution $w$, which is the time that each bead spends in the pore during the translocation process. In the IFTP theory, to obtain the WT we need to calculate the inverse of $\tilde{s}(t)$, i.e., $t(\tilde{s})$. Then the WT is obtained as $w(\widetilde{s})=t(\tilde{s}+$ $0.5)-t(\tilde{s}-0.5)$. On the other hand, in the LD simulations the WT can be obtained directly by recording the time that each bead spends in the pore during the translocation process. In Fig. 4(a) we plot $w(\tilde{s})$ as a function of the total translocation coordinate $\tilde{s}=\tilde{s}_{1}+\tilde{s}_{2}\left(\tilde{s}_{1}\right.$ and $\tilde{s}_{2}$ correspond to the cisand trans-side subchains, respectively) for $N_{02}=100, F_{\mathrm{SP}}=$ $32, L_{\mathrm{r}}=16$, the pore friction coefficient in the IFTP theory $\tilde{\eta}_{\mathrm{p}}=8$ (which can be obtained by comparing the WT from IFTP with LD simulations and once it has been obtained it is fixed), and for different values of the initial cis-side contour lengths $N_{01}=50$ (open turquoise squares), $N_{01}=100$ (open green circles) and $N_{01}=150$ (open orange diamonds). The solid blue, dashed green, and dash-dotted red lines present the IFTP results for $N_{01}=50,100$, and 150, respectively. Regions with $0<\tilde{s} \leqslant N_{02}$ and $N_{02}<\tilde{s} \leqslant N_{0}$ identify the monomers initially in the trans- and the cis-side subchains, respectively. Here $N_{02}=100$ has been fixed in order to have the same initial configuration for the trans-side subsystem. This allows us to investigate only the effect of the initial contour length of the cis-side subchain on the translocation process. We find good agreement between the LD simulation results and the IFTP theory. The simulation data show that the trans-side subchain $\left(0<\tilde{s} \leqslant N_{02}\right)$ contributes to the WT (see Fig. 4) due to the small magnitude of $\tilde{f}(\tilde{t})$ at the beginning of translocation 


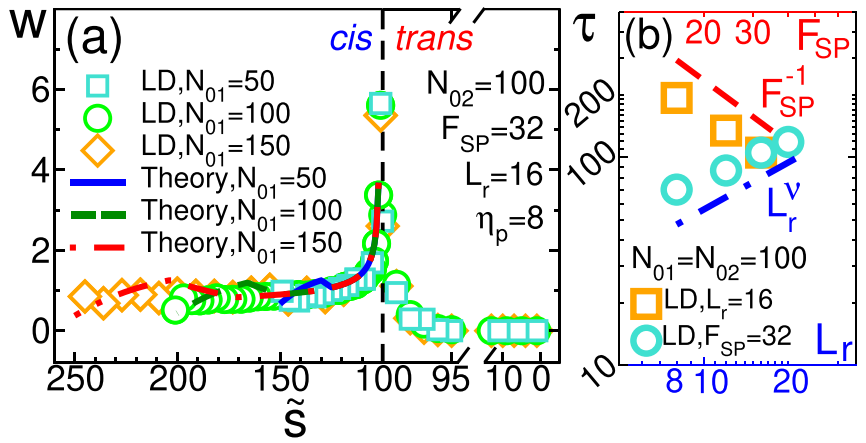

FIG. 4. (a) Waiting time $w$ as a function of the total translocation coordinate $\tilde{s}$ for fixed values of the initial trans-side contour length $N_{02}=100$, SP force $F_{\mathrm{SP}}=32$, rod length $L_{\mathrm{r}}=16$, and pore friction in the IFTP theory $\eta_{\mathrm{p}}=8$ and for different values of the initial cisside contour lengths $N_{01}=50$ (open turquoise squares), $N_{01}=100$ (open green circles), and $N_{01}=150$ (open orange diamonds). The solid blue, dashed green, and red dash-dotted lines are the IFTP results for $N_{01}=50,100$, and 150, respectively. (b) Translocation time $\tau$ (from LD simulations) plotted as a function of rod length $L_{\mathrm{r}}$ for fixed $F_{\mathrm{SP}}=32$ (open turquoise circles from the bottom horizontal blue axis). Open orange squares are the LD data for the translocation time as a function of $F_{\mathrm{SP}}$ (from the top horizontal red axis) for fixed $L_{\mathrm{r}}=16$. Dashed red and dash-dotted blue lines are guides to the eye.

process because a short section of the trans-side subchain is temporarily retracted to the cis side. The IFTP theory thus slightly underestimates the WT as it considers the dynamics of the cis-side subchain only.

\section{B. Scaling of translocation time}

Next we consider the average translocation time $\tilde{\tau}$ for the polymer needs to pass through the nanopore. Its scaling form can be written as $\tilde{\tau} \propto \tilde{F}_{\mathrm{SP}}^{\beta} \tilde{L}_{\mathrm{r}}^{\gamma} N_{01}^{\alpha}$, where $\alpha, \beta$, and $\gamma$ are the translocation, SP force, and rod length exponents, respectively. Combining mass conservation in the TP and PP stages, i.e., $N_{1}=\tilde{s}_{1}+\tilde{l}_{1}$ and $N_{1}=\tilde{s}_{1}+\tilde{l}_{1}=N_{01}$, respectively, with Eq. (1), the TP time is obtained by integration of $N_{1}$ from zero to $N_{01}$, while the PP time is calculated by integration of $\tilde{R}_{1}$ from $\tilde{R}_{1}\left(N_{01}\right)$ to zero [24]. The sum of the TP and PP times leads to

$$
\int_{0}^{\tilde{t}_{\mathrm{TP}}} \tilde{f}_{\mathrm{TP}}(\tilde{t}) d \tilde{t}+\int_{\tilde{t}_{\mathrm{TP}}}^{\tilde{\tau}} \tilde{f}_{\mathrm{PP}}(\tilde{t}) d \tilde{t}=\int_{0}^{N_{01}} d N_{1}\left[\tilde{R}_{1}\left(N_{1}\right)+\tilde{\eta}_{\mathrm{p}}\right],
$$

where the effective forces in the TP and PP stages of this relation are obtained from fitting to the simulation data in Fig. 2(a) for TP (solid green line) and PP (dash-dotted green line) stages, respectively, as

$$
\begin{aligned}
& \tilde{f}_{\mathrm{TP}}(\tilde{t}) \tilde{L}_{\mathrm{r}}^{v} / \tilde{F}_{\mathrm{SP}}=a+b \tilde{t} / \tilde{\tau}, \\
& \tilde{f}_{\mathrm{PP}}(\tilde{t}) \tilde{L}_{\mathrm{r}}^{v} / \tilde{F}_{\mathrm{SP}}=c-d \tilde{t} / \tilde{\tau},
\end{aligned}
$$

with $a=0.35, b=13.25, c=11.37$, and $d=6.42$. Using $\tilde{f}_{\mathrm{TP}}\left(\tilde{t}=\tilde{t}_{\mathrm{TP}}\right)=\tilde{f}_{\mathrm{PP}}\left(\tilde{t}=\tilde{t}_{\mathrm{TP}}\right)$, the TP time is then given by $\tilde{t}_{\mathrm{TP}}=Q \tilde{\tau}$, where $Q=(c-a) /(b+d) \approx 0.56$ here, as mentioned earlier. Combining $\tilde{t}_{\mathrm{TP}}=Q \tilde{\tau}$ with the effective forces in the TP and PP stages in Eq. (5) together with Eq. (4) gives the total translocation time as

$$
\tilde{\tau}=\tilde{L}_{\mathrm{r}}^{\nu} \int_{0}^{N_{01}} d N_{1}\left[\tilde{R}_{1}\left(N_{1}\right)+\tilde{\eta}_{\mathrm{p}}\right] / G \tilde{F}_{\mathrm{SP}}
$$

Using $\tilde{R}_{1}\left(N_{1}\right)=A_{v} N_{1}^{v}$, where $v=3 / 4$ in $2 \mathrm{D}$ and $A_{v}=1.1$ (from LD data), the scaling of the translocation time is

$$
\tilde{\tau}=\frac{\tilde{L}_{\mathrm{r}}^{v}}{G \tilde{F}_{\mathrm{SP}}}\left[\frac{A_{v} N_{01}^{1+v}}{1+v}+N_{01} \tilde{\eta}_{\mathrm{p}}\right]
$$

where $G=-0.5(c-a)^{2} /(b+d)+c-d / 2 \approx 11.25$. Equation (7) reveals that the SP force and rod length exponents are $\beta=-1$ and $\gamma=\nu$, respectively, and the translocation exponent varies within $1<\alpha \leqslant 1+v$, where the upper bound holds in the long-chain limit $N_{01} \gg 1$. The SP force and translocation exponents are in agreement with purely poredriven translocation in the SS regime [24]. In Fig. 4(b) we plot the translocation time as a function of the SP force (open orange squares from the top red horizontal axis) and the rod length (open turquoise circles from the bottom blue horizontal axis). Dashed red and dash-dotted blue lines are guides to the eye. The SP force and rod length exponents obtained from IFTP theory are in good agreement with the LD data.

\section{CONCLUSION}

In summary, we have shown here that active rodlike particles on the trans side of a membrane can efficiently overcome entropic losses and facilitate translocation of a polymer chain through a nanopore. The SP force induces a crowding effect of the rods close to the membrane and the polymer, and as a net result there is an effective driving force making translocation possible even without explicit driving. We have used a combination of LD simulations and IFTP theory in the SS regime to characterize the waiting time distribution $w$ and the average translocation time $\tau$. Neglecting the explicit contribution of $N_{02}$ to the dynamics allowed us to derive a scaling form for $\tilde{\tau}$ as a function of $N_{01}$, SP force, and rod length. The scaling exponents for $\tau$ and the SP force are in agreement with those of the purely pore-driven translocation case. Here we have only focused on systems wherein the initial values of the contour lengths for the trans-side subchain are long enough to lead to successful translocation events. Moreover, even for the system composed of $50 \%$ of the chain already on the trans side, for a successful translocation there would be a threshold for the value of $\tilde{F}_{\mathrm{SP}}$ in order to overcome the entropic force. On the other hand, at a constant value of $\tilde{F}_{\mathrm{SP}}$, there exists a threshold value for $N_{02} / N_{0}$ in order to overcome the entropic force. For this case the threshold value of the $N_{02}$ depends on the density of the ARs as well as the value of $\tilde{F}_{\text {SP. Our work }}$ provides insight into our knowledge about the role of APs in living cells that may assist translocation of biomolecules and may be used as a method to control translocation dynamics, which is crucial for DNA sequencing applications.

\section{ACKNOWLEDGMENTS}

Computational resources from Center for Scientific Computing Ltd. are gratefully acknowledged. T.A-N. was supported in part by the Academy of Finland through its PolyDyna (Grant No. 307806) and QFT Center of Excellence Program grants (Grant No. 312298). 
[1] M. Muthukumar, Polymer Translocation (Taylor \& Francis, Boca Raton, 2011).

[2] V. V. Palyulin, T. Ala-Nissila, and R. Metzler, Polymer translocation: The first two decades and the recent diversification, Soft Matter 10, 9016 (2014).

[3] A. Milchev, Single-polymer dynamics under constraints: Scaling theory and computer experiment, J. Phys.: Condens. Matter 23, 103101 (2011).

[4] J. Sarabadani and T. Ala-Nissila, Theory of pore-driven and end-pulled polymer translocation dynamics through a nanopore: An overview, J. Phys.: Condens. Matter 30, 274002 (2018).

[5] B. Alberts, A. Johnson, J. Lewis, M. Raff, K. Roberts, and P. Walter, Molecular Biology of the Cell (Garland Science, New York, 2002).

[6] A. Meller, L. Nivon, and D. Branton, Voltage-Driven DNA Translocations through a Nanopore, Phys. Rev. Lett. 86, 3435 (2001).

[7] D. E. Smith, S. J. Tans, S. B. Smith, S. Grimes, D. L. Anderson, and C. Bustamante, The bacteriophage $\varphi 29$ portal motor can package DNA against a large internal force, Nature (London) 413, 748 (2001).

[8] A. J. Storm, J. H. Chen, X. S. Ling, H. W. Zandbergen, and C. Dekker, Fabrication of solid-state nanopores with singlenanometre precision, Nat. Mater. 2, 537 (2003).

[9] A. F. Sauer-Budge, J. A. Nyamwanda, D. K. Lubensky, and D. Branton, Unzipping Kinetics of Double-Stranded DNA in a Nanopore, Phys. Rev. Lett. 90, 238101 (2003).

[10] A. J. Storm, C. Storm, J. Chen, H. Zandbergen, J.-F. Joanny, and C. Dekker, Fast DNA translocation through a solid-state nanopore, Nano Lett. 5, 1193 (2005).

[11] U. F. Keyser, B. N. Koeleman, S. van Dorp, D. Krapf, R. M. M. Smeets, S. G. Lemay, N. H. Dekker, and C. Dekker, Direct force measurements on DNA in a solid-state nanopore, Nat. Phys. 2, 473 (2006).

[12] U. F. Keyser, N. H. Dekker, C. Dekker, and S. G. Lemay, Origin of the electrophoretic force on DNA in solid-state nanopores, Nat. Phys. 5, 347 (2009).

[13] R. D. Bulushev, S. Marion, and A. Radenovic, Relevance of the drag force during controlled translocation of a DNA-protein complex through a glass nanocapillary, Nano Lett. 15, 7118 (2015).

[14] W. Sung and P. J. Park, Polymer Translocation through a Pore in a Membrane, Phys. Rev. Lett. 77, 783 (1996).

[15] M. Muthukumar, Polymer translocation through a hole, J. Chem. Phys. 111, 10371 (1999).

[16] Y. Kantor and M. Kardar, Anomalous dynamics of forced translocation, Phys. Rev. E 69, 021806 (2004).

[17] A. Y. Grosberg, S. Nechaev, M. Tamm, and O. Vasilyev, How Long Does It Take to Pull an Ideal Polymer into a Small Hole? Phys. Rev. Lett. 96, 228105 (2006).

[18] G. Sigalov, J. Comer, G. Timp, and A. Aksimentiev, Detection of DNA sequences using an alternating electric field in a nanopore capacitor, Nano Lett. 8, 56 (2008).

[19] P. Rowghanian and A. Y. Grosberg, Force-driven polymer translocation through a nanopore: An old problem revisited, J. Phys. Chem. B 115, 14127 (2011).

[20] T. Sakaue, Nonequilibrium dynamics of polymer translocation and straightening, Phys. Rev. E 76, 021803 (2007).

[21] T. Sakaue, Phys. Rev. E 81, 041808 (2010).
[22] T. Saito and T. Sakaue, Process time distribution of driven polymer transport, Phys. Rev. E 85, 061803 (2012).

[23] T. Ikonen, A. Bhattacharya, T. Ala-Nissila, and W. Sung, Unifying model of driven polymer translocation, Phys. Rev. E 85, 051803 (2012).

[24] J. Sarabadani, T. Ikonen, and T. Ala-Nissila, Iso-flux tension propagation theory of driven polymer translocation: The role of initial configurations, J. Chem. Phys. 141, 214907 (2014).

[25] J. Sarabadani, T. Ikonen, and T. Ala-Nissila, Theory of polymer translocation through a flickering nanopore under an alternating driving force, J. Chem. Phys. 143, 074905 (2015).

[26] J. Sarabadani, B. Ghosh, S. Chaudhury, and T. Ala-Nissila, Dynamics of end-pulled polymer translocation through a nanopore, Europhys. Lett. 120, 38004 (2017).

[27] J. Sarabadani, T. Ikonen, H. Mökkönen, T. Ala-Nissila, S. Carson, and M. Wanunu, Driven translocation of a semi-flexible polymer through a nanopore, Sci. Rep. 7, 7423 (2017).

[28] H. W. de Haan and G. W. Slater, Mapping the variation of the translocation $\alpha$ scaling exponent with nanopore width, Phys. Rev. E 81, 051802 (2010).

[29] H. W. de Haan and G. W. Slater, Using an incremental mean first passage approach to explore the viscosity dependent dynamics of the unbiased translocation of a polymer through a nanopore, J. Chem. Phys. 136, 204902 (2012).

[30] M. G. Gauthier and G. W. Slater, Nondriven polymer translocation through a nanopore: Computational evidence that the escape and relaxation processes are coupled, Phys. Rev. E 79, 021802 (2009).

[31] N. Nikoofard, H. Khalilian, and H. Fazli, Directed translocation of a flexible polymer through a cone-shaped nano-channel, J. Chem. Phys. 139, 074901 (2013).

[32] S. Buyukdagli, J. Sarabadani, and T. Ala-Nissila, Dielectric trapping of biopolymers translocating through insulating membranes, Polymers 10, 1242 (2018).

[33] S. Buyukdagli, J. Sarabadani, and T. Ala-Nissila, Theoretical modeling of polymer translocation: From the electrohydrodynamics of short polymers to the fluctuating long polymers, Polymers 11, 118 (2019).

[34] J. Sarabadani, S. Buyukdagli, and T. Ala-Nissila, Pulling a DNA molecule through a nanopore embedded in an anionic membrane: Tension propagation coupled to electrostatics, J. Phys.: Condens. Matter 32, 385101 (2020).

[35] B. Ghosh, J. Sarabadani, S. Chaudhury, and T. Ala-Nissila, Pulling a folded polymer through a nanopore, J. Phys.: Condens. Matter 33, 015101 (2020).

[36] J. A. Cohen, A. Chaudhuri, and R. Golestanian, Active Polymer Translocation through Flickering Pores, Phys. Rev. Lett. 107, 238102 (2011).

[37] T. Menais, S. Mossa, and A. Buhot, Polymer translocation through nano-pores in vibrating thin membranes, Sci. Rep. 6, 38558 (2017).

[38] T. Menais, Polymer translocation under a pulling force: Scaling arguments and threshold forces, Phys. Rev. E 97, 022501 (2018).

[39] J.-W. Yeh, A. Taloni, Y.-L. Chen, and C.-F. Chou, Entropydriven single molecule tug-of-war of DNA at micro-nanofluidic interfaces, Nano Lett. 12, 1597 (2012).

[40] R. Adhikari and A. Bhattacharya, Translocation of a semiflexible polymer through a nanopore in the presence of attractive binding particles, Phys. Rev. E 92, 032711 (2015). 
[41] A. Bhattacharya and S. Seth, Tug of war in a double-nanopore system, Phys. Rev. E 101, 052407 (2020).

[42] S. M. Bezrukov, I. Vodyanoy, and A. V. Parsegian, Counting polymers moving through a single ion channel, Nature (London) 370, 279 (1994).

[43] J. J. Kasianowicz, E. Brandin, D. Branton, and D. W. Deamer, Characterization of individual polynucleotide molecules using a membrane channel, Proc. Natl. Acad. Sci. USA 93, 13770 (1996).

[44] A. Gopinathan and Y. W. Kim, Polymer Translocation in Crowded Environments, Phys. Rev. Lett. 99, 228106 (2007).

[45] Y. Chen and K. Luo, Dynamics of polymer translocation through a nanopore induced by different sizes of crowding agents, J. Chem. Phys. 138, 204903 (2013).

[46] W. Yu and K. Luo, Polymer translocation through a nanopore driven by binding particles: Influence of chain rigidity, Phys. Rev. E 90, 042708 (2014).

[47] F. S. Taheri, H. Fazli, M. Doi, and M. Habibi, Granular chain escape from a pore in a wall in the presence of particles on one side: A comparison to polymer translocation, Soft Matter 14, 5420 (2018).

[48] R. H. Abdolvahab, M. R. Ejtehadi, and R. Metzler, Sequence dependence of the binding energy in chaperone-driven polymer translocation through a nanopore, Phys. Rev. E 83, 011902 (2011).

[49] S. Emamyari and H. Fazli, Polymer translocation through a nanopore in the presence of chaperones: A three dimensional MD simulation study, Comput. Condens. Matter 13, 96 (2017).

[50] X. Xu and Y. Zhang, Mean velocity and effective diffusion constant for translocation of biopolymer chains across membrane, J. Stat. Mech. (2020) 023501.

[51] T. Vicsek, A. Czirok, E. Ben-Jacob, I. Cohen, and O. Shochet, Novel Type of Phase Transition in a System of Self-Driven Particles, Phys. Rev. Lett. 75, 1226 (1995).

[52] T. Vicsek and A. Zafeiris, Collective motion, Phys. Rep. 517, 71 (2012).

[53] E. R. Kay, D. A. Leigh, and F. Zerbetto, Synthetic molecular motors and mechanical machines, Angew. Chem. Int. Ed. 46, 72 (2007).

[54] C. A. Weber, T. Hanke, J. Deseigne, S. Leonard, O. Dauchot, E. Frey, and H. Chate, Long-Range Ordering of Vibrated Polar Disks, Phys. Rev. Lett. 110, 208001 (2013).

[55] H. Berg, Random Walks in Biology (Princeton University, Princeton, 1983).

[56] A. Okubo and S. Levin, Diffusion and Ecological Problems (Springer, New York, 1980).

[57] R. Dreyfus, J. Baudry, M. L. Roper, M. Fermigier, H. A. Stone, and J. Bibette, Microscopic artificial swimmers, Nature (London) 437, 862 (2005).

[58] M. Leoni, J. Kotar, B. Bassetti, P. Cicuta, and M. C. Lagomarsino, A basic swimmer at low Reynolds number, Soft Matter 5, 472 (2009).

[59] E. M. Purcell, Life at low Reynolds number, Am. J. Phys. 45, 3 (1977).

[60] J. E. Avron, O. Kenneth, and D. H. Oaknin, Pushmepullyou: An efficient micro-swimmer, New J. Phys. 7, 234 (2005).
[61] R. Golestanian and A. Ajdari, Mechanical Response of a Small Swimmer Driven by Conformational Transitions, Phys. Rev. Lett. 100, 038101 (2008).

[62] J. Deseigne, O. Dauchot, and H. Chate, Collective Motion of Vibrated Polar Disks, Phys. Rev. Lett. 105, 098001 (2010).

[63] J. Deseigne, S. Leonard, O. Dauchot, and H. Chate, Vibrated polar disks: Spontaneous motion, binary collisions, and collective dynamics, Soft Matter 8, 5629 (2012).

[64] D. L. Blair, T. Neicu, and A. Kudrolli, Vortices in vibrated granular rods, Phys. Rev. E 67, 031303 (2003).

[65] V. Narayan, N. Menon, and S. Ramaswamy, Nonequilibrium steady states in a vibrated-rod monolayer: Tetratic, nematic, and smectic correlations, J. Stat. Mech. (2006) P01005.

[66] I. S. Aranson, D. Volfson, and L. S. Tsimring, Swirling motion in a system of vibrated elongated particles, Phys. Rev. E 75, 051301 (2007).

[67] A. Kudrolli, G. Lumay, D. Volfson, and L. S. Tsimring, Swarming and Swirling in Self-Propelled Polar Granular Rods, Phys. Rev. Lett. 100, 058001 (2008).

[68] A. Kudrolli, Concentration Dependent Diffusion of SelfPropelled Rods, Phys. Rev. Lett. 104, 088001 (2010).

[69] L. J. Daniels, Y. Park, T. C. Lubensky, and D. J. Durian, Dynamics of gas-fluidized granular rods, Phys. Rev. E 79, 041301 (2009).

[70] A. Kaiser and H. Löwen, Unusual swelling of a polymer in a bacterial bath, J. Chem. Phys. 141, 044903 (2014).

[71] J. Harder, C. Valeriani, and A. Cacciuto, Activity-induced collapse and reexpansion of rigid polymers, Phys. Rev. E 90, 062312 (2014).

[72] R. Chelakkot, A. Gopinath, L. Mahadevan, and M. F. Hagan, Flagellar dynamics of a connected chain of active, polar, Brownian particles, J. R. Soc. Interface 11, 20130884 (2013).

[73] H. Jiang and Z. Hou, Hydrodynamic interaction induced spontaneous rotation of coupled active filaments, Soft Matter 10, 9248 (2014).

[74] H. Jiang and Z. Hou, Motion transition of active filaments: Rotation without hydrodynamic interactions, Soft Matter 10, 1012 (2014).

[75] X. Liu, H. Jiang, and Z. Hou, Configuration dynamics of a flexible polymer chain in a bath of chiral active particles, J. Chem. Phys. 151, 174904 (2019).

[76] X. Liu, H. Jiang, and Z. Hou, Non-monotonic dependence of polymer chain dynamics on active crowder size, J. Chem. Phys. 152, 204906 (2020).

[77] M. Pu, H. Jiang, and Z. Hou, Polymer translocation through nanopore into active bath, J. Chem. Phys. 145, 174902 (2016).

[78] H. H. Wensink and H. Löwen, Aggregation of self-propelled colloidal rods near confining walls, Phys. Rev. E 78, 031409 (2008).

[79] F. Peruani, A. Deutsch, and M. Bär, Nonequilibrium clustering of self-propelled rods, Phys. Rev. E. 74, 030904(R) (2006).

[80] G. S. Grest and K. Kremer, Molecular dynamics simulation for polymers in the presence of a heat bath, Phys. Rev. A 33, 3628 (1986).

[81] S. Plimpton, Fast parallel algorithms for short-range molecular dynamics, J. Comput. Phys. 117, 1 (1995); http://lammps. sandia.gov. 